\section{Monoklonaler Antikörper als verträglichere Alternative}

Stone JH et al. Rituximab versus Cyclophosphamide for Induction of Remission in ANCA-associated Vasculitis: a randomized controlled trial (RAVE). Arthritis Rheum. 2009;60 Suppl 10:550

\section{Hintergrund}

Die kombinierte Anwendung von Cyclophosphamid und Glukokortikoiden ist seit Jahrzehnten die Standardtherapie zur Induktion einer Remission bei ANCA-assoziierten Vaskulitiden (AAV). Unkontrollierte Studien deuteten schon seit geraumer Zeit darauf hin, dass Rituximab ebenfalls einen Stellenwert in der Therapie von AAV hat, zumal die $\mathrm{Ne}$ benwirkungsrate unter Rituximab erheblich geringer ist als unter Cyclophosphamid [1].

\section{Fragestellung}

Die RAVE-Studie, deren Ergebnisse beim internationalen Rheumatologenkongress in Philadelphia im Oktober 2009 vorgestellt wurden, untersuchte die Wirksamkeit von Rituximab im Vergleich zu Cyclophosphamid in der Behandlung von AAV.

\section{Methodik}

197 Patienten wurden in der multizentrischen, randomisierten, doppelblinden und placebokontrollierten Studie randomisiert, davon 75\% mit einem schweren Morbus Wegener und 25\% mit einer mikroskopischen Polyangiitis. Zu Beginn der Studie erhielten alle Patienten die gleiche Glukokortikoid-Therapie (1-3 g Methylprednisolon i.v., danach Prednison $1 \mathrm{mg} / \mathrm{kg} / \mathrm{d}$ p.o. mit Reduktion auf $40 \mathrm{mg} / \mathrm{d}$ innerhalb eines Monats).

99 Patienten erhielten zusätzlich viermal wöchentlich Rituximab $\left(375 \mathrm{mg} / \mathrm{m}^{2}\right.$, i.v.) und 98 Patienten Cyclophosphamid $(2 \mathrm{mg} / \mathrm{kg} / \mathrm{d}$, oral). Nach Erreichen der Remission wurden die Patienten der Cyclophosphamid-Gruppe auf Azathioprin für drei bis sechs Monate umgestellt.

Primärer Endpunkt war das Erreichen der Remission. Diese wurde definiert als ein Birmingham Vaskultis Acitvity Score for Wegner's granulomatosis (BVAS/ WG) von 0 , bei vollständiger Reduktion des Glukokortikoids.

\section{Ergebnisse}

Nach sechs Monaten hatten 64\% der Patienten aus der Rituximab-Gruppe und 55\% der Patienten aus der Cyclophosphamid-Gruppe eine Remission erreicht. Dieser Unterschied war allerdings nicht signifikant $(\mathrm{p}=0,21)$.

Unterschiede in der Rezidivrate bzw. der Häufigkeit der aufgetretenen Nebenwirkungen waren zwischen den beiden Gruppen nicht zu erkennen, wobei in der Rituximab-Gruppe signifikant weniger Patienten ein unerwünschtes Ereignis hatten (19,2 vs. $32,7 \%, p=0,03)$. Hier spielte vor allem die größere Zahl von Leukopenien in der CyclophosphamidGruppe eine Rolle (16 vs 5).

\section{Schlussfolgerung}

In der Therapie des Morbus Wegener ist Rituximab dem Cyclophosphamid ebenbürtig.

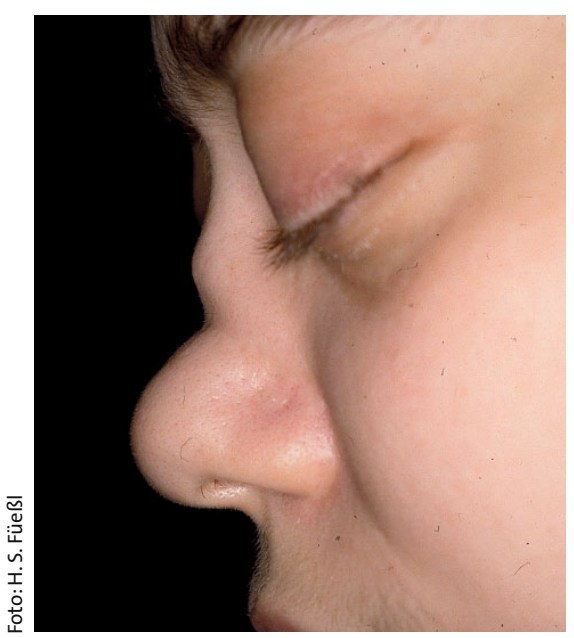

Typische Sattelnase bei einer Patientin mit Morbus Wegener.

\section{Kommentar}

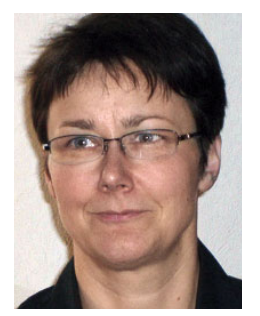

Dr. med.

Kathleen Gutjahr, Leipzig
Rituximab ist ein monoklonaler Antikörper, der 1998 für die Therapie des Non-HodgkinLymphoms und 2006 für die rheumatoide Arthritis zugelassen wurde.

Die Therapie mit Cyclophosphamid ist seit vielen Jahren die einzige Möglichkeit bei schweren Verlaufsformen der AAV bzw. bei Multiorganbefall. Die kumulative Dosis von $50 \mathrm{mg}$ ist gerade bei diesen Patienten schnell erreicht, und die Nebenwirkungsrate ist nicht unerheblich.

Seit einigen Jahren gibt es immer wieder Einzelberichte in der Literatur, die die Wirksamkeit von Rituximab bei cyclophosphamidrefraktären Fällen beschreiben [2]. Umso wichtiger war es, diese Erkenntnisse in einer Studie zu untersuchen.

Die Ergebnisse dieser Studie belegen nun, dass Rituximab eine Therapiealternative zu der bisherigen Standardtherapie mit Cyclophosphamid darstellt.

Trotz der überwiegend positiven Ergebnisse der Studie sollte man nach wie vor auch an mögliche Nebenwirkungen von Rituximab, z.B. Infusionsreaktionen, schwere Infektionen, denken, darüber hinaus auch an das Risiko einer Malignomentwicklung und das möglicherweise gehäufte Auftreten von progressiven multifokalen Leukenzephalopathien.

\footnotetext{
Literatur

1. Wong ChF. Rituximab in refraktory antineutrophil cytoplasmic antibody-associated vasculitis: what is the current evidence. Nephrol Dial Transplant. 2007; 22:32-36

2. Keogh KA. Rituximab for refractory Wegener's Granulomatosis. Am J Respir Crit Care Med. 2006;173: 180-187
} 